Aspirin. We recommend larger studies to interrogate the safety of continuing Aspirin in such patients.

\section{PTU-117 SPLIT DOSE BOWEL PREPARATION: ASSESSING PATIENTS' WILLINGNESS TO WAKE UP EARLY}

AR Shirazi-Nejad*, T Archer, A Al-Rifaie, J Schembri, M Thoufeeq, SA Riley. Department of Gastroenterology, Sheffield Teaching Hospitals, Sheffield, UK

\subsection{6/gutjnl-2019-BSGAbstracts. 106}

Introduction Split dose preparation has been shown to optimise mucosal cleansing, enhance endoscopic views, and improve pathology detection. Split dosing for morning appointments necessitates waking early to take the second dose at an appropriate time. Many units therefore advise that all the preparation is taken the day before the procedure. Our aim was to assess whether patients would be willing to wakeup early to facilitate split dose preparation.

Methods Consecutive patients attending for bowel cancer screening and symptomatic colonoscopy were invited to complete a standardised questionnaire. The results were collated, and logistic regressions were performed in both groups.

Results 418 patients were asked to complete the questionnaire (119 screening and 299 symptomatic); 8 questionnaires were incomplete (6 screening and 2 symptomatic) and were excluded. The M:F ratio was 1.64:1 and 1.03:1; and ages ranged from 55-74 (mean 64.5) and 17-81 (mean 51.5), in the screening and symptomatic groups respectively. Overall, $89 \%$ of screening and $85 \%$ of symptomatic patients reported they would be willing to wake up early to take split-dose preparation for morning appointments. $78 \%$ of screening and $61 \%$ of symptomatic patients would be willing to wake between 4-6 am, to facilitate split dosing for morning scheduled colonoscopies. In the screening patients, 3 factors were found to affect willingness to wake up early: scheduled appointment time, whether they perceived the bowel preparation had a severely unpleasant taste and whether taking the preparation had made them interrupt their journey to the hospital to defaecate.

Conclusions This study indicates that most patients would be willing to wake-up early to take adequately spaced split doses of bowel preparation, irrespective of age, sex, socioeconomic background, indication, or previous colonoscopy. In the screening group, those that felt that the preparation tasted severely unpleasant and those who had to interrupt their journey to hospital to use a toilet, were less likely to be willing to wake early to take bowel preparation. All endoscopy units should be encouraged to invite patients scheduled for morning colonoscopy to wake early to facilitate split dose bowel preparation.

\section{PTU-118 ENDOLUMINAL VACUUM THERAPY FOR THE MANAGEMENT OF BOERHAAVE SYNDROME}

Sophie Stevens*, Robert Thomas, Christopher Peters, Krishna Moorthy, George Hanna, Natalie Direkze, Jonathan Hoare. St. Mary's Hospital, Imperial College Healthcare Trust, London, UK

\subsection{6/gutjnl-2019-BSGAbstracts. 107}

Introduction Boerhaave syndrome is associated with high morbidity and mortality rates. Outcomes are dependent on early recognition and intervention. Until recently, surgery has been the mainstay of management. However, with recent advances in therapeutic endoscopy, there has been increasing interest in endoscopic options, including endoluminal vacuum therapy (EVT). EVT is a minimally invasive technique, allowing wound debridement and drainage; promoting granulation tissue formation to enable wound healing. EVT has been associated with excellent clinical outcomes, including lower mortality rates when compared to both surgery and oesophageal stenting. EVT has been adopted into practice across Europe for the management of oesophageal perforations. However, in the UK there have only been two cases reported. We report a case of a 66 year old female with Boerhaave syndrome, successfully managed with EVT.

Methods EVT for oesophageal perforation involves the placement of a polyurethane sponge into the wound cavity. The cavity is initially assessed with an endoscope before an overtube is introduced under visual control. The sponge is pushed into the cavity through the overtube. Once the sponge is in place, the overtube is removed; allowing the sponge to unfold. Sponge position is confirmed endoscopically and adjusted if necessary. The sponge is connected via a trans-nasal drain to continuous negative pressure. Sponge exchange is performed every 3-5 days.

Results Having been deemed unfit for surgical intervention, due to difficulties with ventilation and haemodynamic instability, our patient was initially stabilised on the Intensive Care Unit (ICU) with the aid of radiologically placed chest drains and intravenous antibiotics. She was subsequently referred for EVT, using the EsoSPONGE ${ }^{\circledR}$ (B.Braun, Medical Ltd, Sheffield, UK). Her first therapeutic endoscopy took place on Day 10 of admission; revealing a $5 \mathrm{~cm}$ defect in the oesophageal wall and an adjacent cavity; major vessels and the chest drains could be visualised endoscopically. The EsoSPONGE was placed into the cavity and connected to suction. She remained on ICU for 3 months and during this period her EsoSPONGE was exchanged 16 times; resulting in resolution of sepsis and healing of the defect. She was successfully stepped down from ICU and has now been discharged.

Conclusions EVT was an effective management strategy for our patient with Boerhaave syndrome. Use of the EsoSPONGE aided drainage of the septic focus and closure of the defect; with this our patient made an excellent recovery. Mortality for this case would otherwise have been extremely high as she was too unstable for surgery. This case supports the evidence that EVT provides a promising approach for the management of Boerhaave syndrome.

\section{PTU-119 ROBOT CONTROLLED MAGNET-ASSISTED CAPSULE GASTROSCOPY IS BETTER TOLERATED AND ACCEPTED BY PATIENTS THAN FLEXIBLE GASTROSCOPY}

David Tai* ${ }^{*}$ Hey Ching, Mark McAlindon. Sheffield Teaching Hospitals, Sheffield, UK

\subsection{6/gutjnl-2019-BSGAbstracts. 108}

Introduction OGD is performed commonly but often poorly tolerated. Capsule endoscopy is well tolerated. Robot controlled magnet assisted capsule endoscopy (MACE) allows control of a capsule endoscope resulting in a non-invasive endoscopic examination of the upper GI tract. No controlled comparisons of patient tolerance and acceptability between MACE and OGD have been performed. Post procedure Endoscopy Concerns Scale (ECS) scores correlate with patient 
acceptance after OGD [Condon 2008, Can J Gastro]. We assess tolerance and acceptability of MACE compared to OGD using the ECS.

Methods Patients referred for the endoscopic investigation of dyspepsia were recruited. Upper GI MACE examinations were performed followed by OGD. Questionnaires examined patients' tolerance to and acceptability of the endoscopies. Paired differences between median distress and ECS scores are reported $\left(\mathrm{p}<0.0001^{* * *}, \mathrm{p}<0.01^{* * *}\right)$.

Results Thirty eight (oral $\mathrm{n}=33$, transnasal $\mathrm{n}=5$ ) patients were recruited (median age 50, female 74\%, sedated in $36.4 \%$ of oral OGDs). All patients tolerated MACE adequately with minimal discomfort in $16 \%$. OGD was adequately tolerated in $63 \%$ with the remaining reporting moderate or severe discomfort. There was less distress ( 1 - 10: not at all - extremely) caused by: discomfort during ( 1 vs $5 * * *)$, discomfort after (1 vs $2 * * *)$ and pain during (1 vs $2 * * *)$ the procedures, gagging (1 vs $\left.6^{* * *}\right)$, choking ( 1 vs $\left.4.5^{* * *}\right)$, bloating ( 1 vs $2^{* *}$ ) and swallowing the capsule vs. insertion of the scope (1 vs $4 * * *)$ with MACE compared to OGD. The overall pre-procedure ECS score ( 28 vs 50 points $* * *)$ and post-procedure ECS score (13 vs 32 points***) was lower after MACE compared to OGD suggesting patients are more accepting of MACE before and after the procedure compared to OGD. There was a reduction in pre-procedure ECS after both MACE (median difference $-15 * *)$ and OGD $(-18 * *)$ suggesting procedures are not as distressing as initially anticipated, however $32 \%$ of patients report a higher ECS after gastroscopy whereas all patients reported a lower ECS after MACE. Patient preference towards MACE or OGD illustrated in table 1 below.

\begin{tabular}{|c|c|c|c|}
\hline & MACE & OGD & \\
\hline Patient preference & Yes, n (\%) & & $\mathrm{p}$ \\
\hline $\begin{array}{l}\text { Undergo the same test under the same } \\
\text { medical circumstances? }\end{array}$ & $38(100)$ & $30(79.0)$ & $* * *$ \\
\hline $\begin{array}{l}\text { Advise a friend to undergo the same test } \\
\text { given the same medical circumstances }\end{array}$ & $38(100)$ & $27(71.0)$ & $* * *$ \\
\hline $\begin{array}{l}\text { As a screening for cancer in } 1 \text { to } 2 \text { years } \\
\text { time? }\end{array}$ & $38(100)$ & $30(79.0)$ & $* * *$ \\
\hline $\begin{array}{l}\text { If the chance of requiring biopsy after } \\
\text { MACE was: }\end{array}$ & MACE first & OGD first & \\
\hline 1 in 20 & $10(91)$ & $1(9)$ & \\
\hline 1 in 10 & $10(91)$ & $1(9)$ & \\
\hline 1 in 5 & $9(82)$ & $2(18)$ & \\
\hline 1 in 4 & $8(73)$ & $3(27)$ & \\
\hline 1 in 2 & $8(73)$ & $3(27)$ & \\
\hline
\end{tabular}

Conclusions Patients better tolerate, are more accepting of and prefer MACE over OGD. Where biopsies are required, patients would still prefer capsule endoscopy initially. Cost effectiveness of this approach should be examined

\section{PTU-120 ROBOT CONTROLLED MAGNET-ASSISTED CAPSULE GASTROSCOPY PROVIDES NON-INFERIOR VIEWS TO CONVENTIONAL GASTROSCOPY: A PILOT STUDY}

David Tai* ${ }^{*}$ Hey Long Ching, Mark McAlindon. Sheffield Teaching Hospitals, Sheffield, UK

10.1136/gutjn--2019-BSGAbstracts.109
Introduction Oesophagogastro duodenoscopy (OGD) is undertaken by $1 \%$ of UK population per year but is often poorly tolerated. Capsule endoscopy is well tolerated. Robot controlled magnet assisted capsule endoscopy (MACE) allows control of a capsule endoscope within a pool of swallowed water resulting in a non-invasive endoscopic examination of the upper GI tract. We examine whether there is a difference in the endoscopic views between MACE and OGD.

Methods Patients referred for the endoscopic investigation of dyspepsia were recruited. Upper GI MACE examinations were performed followed by OGD. Endoscopic views of upper GI landmarks on MACE and video recordings of tandem OGDs are graded ( 0 - no views, 1- Poor: $>75 \%$ obscured, 2- Suboptimal: $>50 \%$ obscured, 3- Reasonable: $<50 \%$ obscured, 4Good: $<25 \%$ obscured, 5-Excellent: complete view). Obscuration at MACE defined as debris, bubbles, poor image clarity/ illumination, and at OGD as lack of insufflation, patient retching, mucus or debris. Clarity and distension of stomach is graded (1- poor, 2-reasonable, 3- good). Non-parametric paired differences between MACE and OGD were examined $\left(\mathrm{p}<0.005^{* *}, \mathrm{p}<0.05 \%\right)$.

Results Sixteen (peroral $\mathrm{n}=15$, transnasal $\mathrm{n}=2$ ) patients (median age of 49, female 76\%) were included. Mean examination time for MACE was longer at 46.4 minutes compared to conventional gastroscopy of 4.7 minutes $(\mathrm{p}<0.0001)$. No GOJ views were seen on MACE in $38 \%$ $(6 / 17)$. The capsule was steered into D1 in $12 \%(2 / 17)$ with views of duodenum in $76 \%(13 / 17)$. There were no differences in the reported clarity (2.5 vs 2$)$ and distension (3 vs 2) of stomach between MACE and flexible gastroscopy $(\mathrm{p}<0.05)$. Differences in median endoscopic view scores are show below.

Abstract PTU-120 Table 1 Paired endoscopic view comparisons of MACE and fibre optic gastroscopy

\begin{tabular}{|c|c|c|c|}
\hline Landmark & MACE & OGD & $p$ \\
\hline & Median (IQR) & & \\
\hline Oesophagus & $4.5(2)$ & $5(0)$ & * \\
\hline GOJ & $3(5)$ & $5(1)$ & ** \\
\hline \multirow[t]{2}{*}{ Cardia } & 5 & 4 & \\
\hline & $(0.75)$ & $(1.75)$ & \\
\hline \multirow[t]{2}{*}{ Fundus } & $5(0)$ & 4 & * \\
\hline & & $(2.75)$ & \\
\hline \multirow[t]{2}{*}{ Greater Curve } & 5 & $3.5(1)$ & ** \\
\hline & $(0.75)$ & & \\
\hline Lesser Curve & $5(0)$ & $4(2)$ & * \\
\hline \multirow[t]{2}{*}{ Anterior Body } & 5 & $4(1)$ & ** \\
\hline & $(0.75)$ & & \\
\hline Posterior & $5(1)$ & 4 & ** \\
\hline Body & & $(1.75)$ & \\
\hline Antrum & $5(0)$ & $5(0)$ & \\
\hline D1 & $3(2)$ & $5(0)$ & ** \\
\hline D2 & $4(1)$ & $5(0)$ & \\
\hline
\end{tabular}

Conclusions Unblinded comparisons suggest gastric landmark views are excellent and compared to swifter gastroscopy studies, may better visualise the body and fundus of the stomach. On the other hand, oesophageal and D1 views are superior with OGD and active transpyloric passage into the duodenum using the magnetic fields was limited. This endoscopic view score needs validation by blinded independent parties. Further 